\title{
La visualización del tiempo: de los sistemas de información geográfica a la animación cartográfica
}

\author{
Florian Hruby, María del Rocío Castillo Aja \\ y Luis Valdivia Ornelas
}

\section{Resumen}

El trabajo propuesto pretende discutir el potencial de visualizaciones del tiempo desde una perspectiva teórica y práctica. Las preguntas teóricas abarcan las características del tiempo, la ampliación de las variables visuales de Bertin (1967) con las variables dinámicas de DiBiase et al. (1992) y una tipología de animaciones para estructurar el factor "tiempo" exhaustivamente. A partir de estas bases teóricas podemos analizar, cómo los Sistemas de Información Geográfica (SIG) permiten actualmente la visualización de datos temporales. Sin embargo, este análisis no se limita en una descripción de avances y limitaciones de los SIG, sino que intenta mostrar cómo datos espaciales exportados desde un SIG pueden ser enriquecidos mediante el tratamiento con software de autoría multimedia para la realización de visualizaciones animadas de procesos dinámicos. La actualización del Atlas de riesgos por fenómenos naturales del Estado de Jalisco se usará para ilustrar aspectos tanto teóricos como prácticos del presente artículo.

Palabras clave: Cartografía. Animación. Tiempo. SIG. Atlas

\begin{abstract}
In this article, we attempt to discuss the potential of visualizations of time from a theoretical and practical point of view. Theoretical issues that will concern us include the general characteristics of time, the extension of Bertin's (1967) visual variables by the dynamic variables of DiBiase et al. (1992) and a typology of cartographic animations in order to structure the time-factor in a comprehensive manner. Based on these theoretical fundamentals we will analyze the abilities of current geographic information systems (GIS) to visualize temporal data. In doing so, we won't just focus on the advances and limitations of current GIS, but also outline how geodata exported from the GIS may be enriched in multimedia authoring software so that
\end{abstract}


animations of dynamic processes can be built. The actualization of the Atlas on the risk of natural phenomena in Jalisco will give occasion to illustrate both practical and theoretical aspects in the present contribution.

Keywords: Cartography. Animation. Time. GIS. Atlas.

Florian Hruby (florian@hruby.fastmail.fm), Departamento de Geografía y Ordenación Territorial, Universidad de Guadalajara. María del Rocío Castillo Aja (rocasaja@yahoo.com), Departamento de Geografía y Ordenación Territorial, Universidad de Guadalajara. Luis Valdivia Ornelas (Ivaol2003@yahoo.com.mx), Departamento de Geografía y Ordenación Territorial, Universidad de Guadalajara. 


\section{INTRODUCCIÓN}

El tema de este artículo es la visualización dinámica del tiempo. Teniendo en cuenta la complejidad del término "tiempo" es necesario acotar el concepto; por lo que el concepto tiempo hará referencia a aquello que describe cualquier cambio en nuestro entorno. Ya que no podemos observar el tiempo de manera directa, pero si podemos observar los cambios; en otras palabras: los cambios solo pueden realizarse si el tiempo está transcurriendo. Así queremos en esta contribución utilizar (y visualizar) el tiempo como marco conceptual para describir cambios en las entidades espaciales. Capturar, analizar y visualizar cambios tiene una importancia fundamental para muchas problemas que enfrentan las geociencias actualmente, como por ejemplo el monitoreo y la previsión de riesgos naturales, que requieren un entendimiento de sistemas físicos dinámicos.

La visualización de procesos dinámicos con medios estáticos ha sido un problema en diversas culturas desde hace siglos. Ejemplos clásicos son el Lienzo de Zacatepec 1 (elaborado a mitad del siglo XVI) que contiene información histórica y genealógica del señorío de Zacatepec de aproximadamente 500 años (cf. Mundy, 1996), o el mapa sobre la campaña rusa del ejército napoleónico (Figura 1), publicado en 1869 por Charles Minard (cf. Kraak \& Ormeling, 2010).

Figura 1. La campaña rusa de Napoleón (1812-1813) representada en un mapa de Charles Minard, que muestra el movimiento, las pérdidas humanas y la temperatura ambiental ejemplo de una visualización de tiempo con medios estáticos.

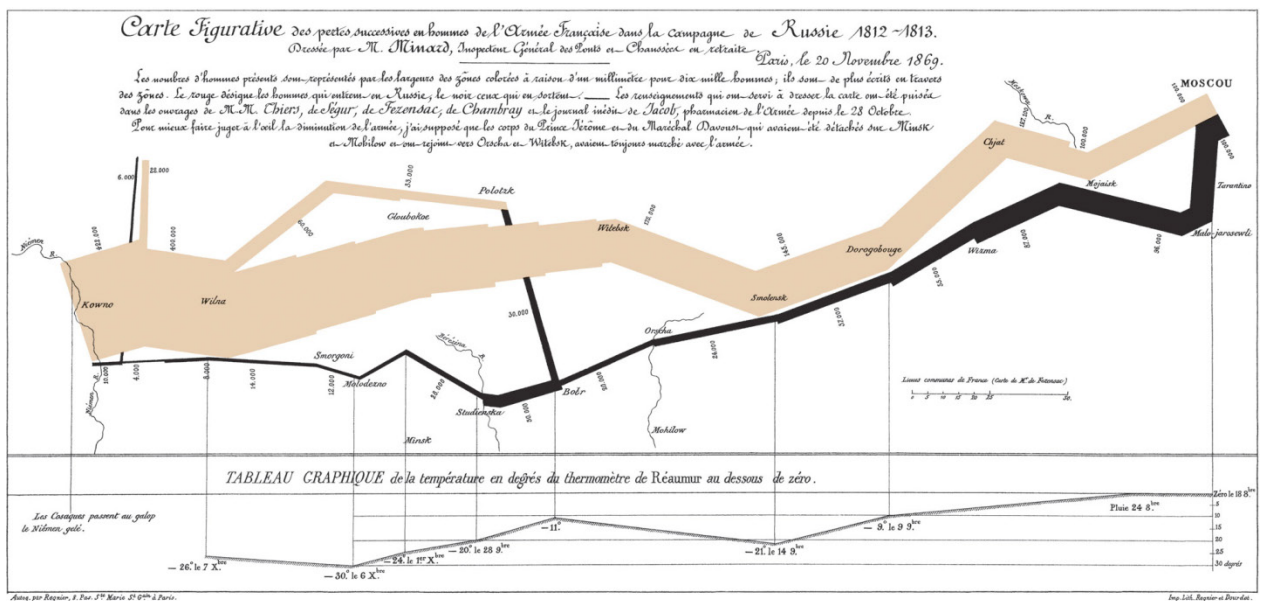

Fuente: Wikimedia Commons; material en dominio público 
La característica común entre todas visualizaciones estáticas de procesos dinámicos es la necesidad de representar el factor tiempo mediante el espacio disponible en el mapa. Puesto que el espacio para la representación cartográfica es limitado, se reducen las opciones para visualizar procesos dinámicos, por lo que sólo es posible presentar uno o algunos puntos del tiempo real. La alternativa para visualizar el tiempo real de manera genuina, haciendo corresponder la variable temporal del fenómeno con la variable tiempo en la representación, se conoce en la cartografía desde Tobler (1970). Sin embargo, estas técnicas no se popularizaron hasta que software y hardware económicos y baratos estuvieron disponibles en los años noventa; en tanto que casi no se realizaron ejemplos de animaciones cartográficas durante los años setenta y ochenta. Al mismo tiempo aumentó el conocimiento empírico sobre las opciones para conseguir un mejor entendimiento de los fenómenos a partir de visualizaciones dinámicas: Por ejemplo observaron Griffin et al. (2006) en una investigación empírica que los participantes en el estudio lograron identificar clusters más rápida y precisamente en representaciones dinámicas que en representaciones estáticas, mientras, Dorling y Openshaw (1992), usando animaciones, encontraron concentraciones (tanto en el espacio como en el tiempo) de casos de leucemia en el norte de Inglaterra, que habían sido desconocidas anteriormente.

Por lo tanto se intentará en los siguientes capítulos, en primer lugar, exponer las características particulares de datos temporales, para poder mostrar, en segundo lugar, cómo estas particularidades pueden ser implementadas tanto dentro de los Sistemas de información Geográfica (SIG) como afuera de los mismos.

\section{EL TIEMPO}

Sin ocuparnos en este articulo de las reflexiones que hacen la física y la filosofía sobre el concepto de tiempo se pueden desprender de la literatura geográfica dos tipos fundamentales: el tiempo como dimensión lineal o como dimensión cíclica (Harrower \& Fabrikant, 2008). Considerar el tiempo como dimensión linear implica un avance consecutivo en el eje temporal; mientras el tiempo cíclico describe más bien el carácter recurrente de ciertos fenómenos. Para la geografía el tiempo como dimensión cíclica es de interés particular, puesto que un extenso entendimiento de eventos recurrentes, por ejemplo de sismos, implica la opción de pronosticar repeticiones futuras con mayor exactitud.

Los datos espaciales y temporales presentan las siguientes analogías (cf. Frank, 1994, Ott \& Swiaczny, 2001): Primero, la dimensión continua, podemos en- 
tender el tiempo, igual que el espacio, como una dimensión continua, sin embargo, por razones prácticas, como son la disponibilidad, la captura y la memorización de datos temporales, en muchos casos es necesario discretizar este continuo. Segundo, la escala, las medidas discretas del tiempo se pueden realizar mediante diferentes escalas, dependiendo si se hace simplemente un orden cronológico de eventos (en términos de una relación "antes-después", ordinal) o si se usan intervalos discretos isócronos con nivel de medida de intervalo o de razón. Tercero, la resolución o generalización, cuando se definen los lapsos de tiempo más pequeños para medir y describir el tiempo de manera discreta, definiendo así la resolución temporal, es decir: la exactitud temporal de una animación cartográfica. Los datos con resoluciones temporales diferentes pueden ser conectados mediante métodos de agregación y descomposición (Figura 2a). Cuarto, la escala gráfica, para dar a entender esta resolución temporal a los usuarios de la animación es necesario especificar la escala temporal que define la relación entre tiempo real y tiempo visualizado. Igual que la escala espacial se puede señalar de manera numérica (por ejemplo tendría una animación que muestra cinco años en cinco segundos una escala de 1:31536000 (=1 seg/1 año) o de manera gráfica (Figura 2b).

Figura 2a: Resolución temporal y su modificación mediante los métodos de agregación y descomposición; Figura 2b: Ejemplo de una escala temporal gráfica (describiendo el huracán Kenna, cf. capítulo x/Figura x).

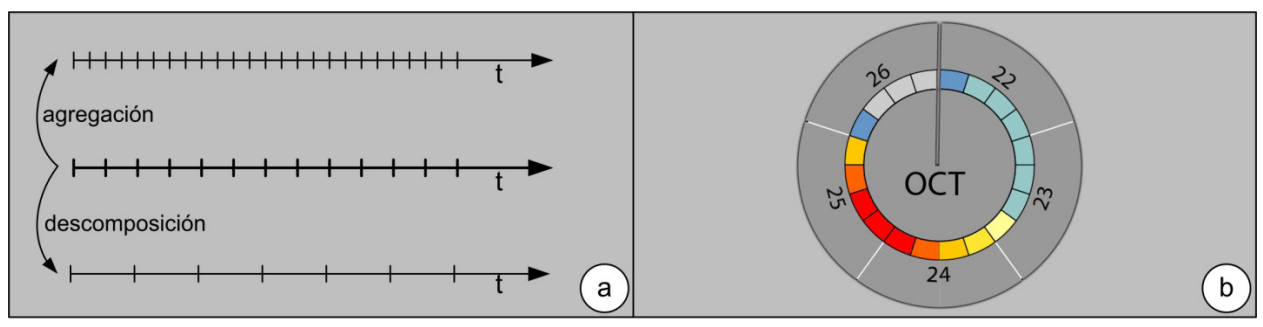

Fuente: Elaboración propia.

\section{VARIABLES ESTÁTICAS Y DINÁMICAS}

A través de la cartografía se pretende visualizar información espacial y temporal de tal manera que permita a los usuarios de productos cartográficos comprender esta información apropiadamente. Para lograr esta meta se utilizan las 6 (+1) variables visuales definidas por Bertin (1967), que describen cómo cualquier símbolo gráfico del mapa se puede modificar, además de la posición (dada por $x, y$ ) en visualizaciones planas por el tamaño, la intensidad o valor, el grano o textura, el 
color, la orientación y la forma. A pesar de que Bertin define las variables visuales explícitamente para visualizaciones estáticas, se pueden visualizar las modificaciones de los valores de estas variables también de manera dinámica. Por ejemplo puede resultar una modificación dinámica de la variable " $x, y$ " de un símbolo del mapa en la percepción de un movimiento continuo de este símbolo por parte del usuario; de la misma manera resultaría la modificación de la variable "orientación" en la percepción de una rotación (Figura 3).

Figura 3. Las variables visuales de Bertin (1967) y sus posibles modificaciones en entornos estáticos y dinámicos

\begin{tabular}{|c|c|c|}
\hline$\frac{\text { VARIABLES GRÁFICAS }}{\text { (BERTIN, 1967) }}$ & $\begin{array}{l}\text { VISUALIZACIONES } \\
\text { ESTATICAS }\end{array}$ & $\begin{array}{l}\text { VISUALIZACIONES } \\
\text { DINAMICAS }\end{array}$ \\
\hline $\begin{array}{l}2 \text { DIMENSIONES } \\
\text { DEL PLANO }\end{array}$ & $y^{\dagger}$ & $y^{\uparrow}$ \\
\hline TAMAÑO & & \\
\hline INTENSIDAD & & \\
\hline GRANO & $\theta$ & \\
\hline COLOR & & \\
\hline ORIENTACIÓN & & \\
\hline FORMA & & \\
\hline
\end{tabular}

Fuente: Elaboración propia.

Para estructurar las opciones que se dan en visualizaciones dinámicas a partir de las variables visuales, DiBiase et al. (1992) propusieren tres variables dinámicas (después modificadas por otros autores, e.g. MacEachren (2004): magnitud de variación (entre dos escenas subsiguientes de una animación; rate of change), duración (duration) y orden (order).

Las visualizaciones estáticas representan normalmente alguna situación 
real en el presente, pasado o futuro, a la cual se refiere en una animación con el término "escena" (DiBiase et al., 1992). Cada animación consta —a diferencia de un mapa estático- de varias escenas, que se pueden describir mediante la variable duración. Esta primera variable dinámica expresa el tiempo que cada escena de la animación permanece visible para el usuario. Dado que el tiempo se puede medir, la duración permite representar datos con niveles de medida (escala) ordinal, de intervalo o razón, por ejemplo para programar la duración de escenas en función de la magnitud del fenómeno representado. Mientras una escena en una animación representa una situación real, una secuencia de situaciones reales representará un evento. Para visualizar un evento mediante una animación, es decir: para visualizar una secuencia de escenas podemos diferenciar, como segunda variable dinámica, la magnitud de la variación; que se podrá expresar mediante la diferencia en la posición o con respecto a un atributo gráfico de un símbolo entre dos escenas consecutivas. Esta variable abre en conjunto con la variable de la duración varias alternativas para diseñar animaciones cartográficas. Dejando, por ejemplo, la magnitud de variación de las variables $x$, y constante y pequeña, y manteniendo constante $y$ pequeña también a la duración, resulta en la sensación de un movimiento continuo para el usuario. Si se aumenta la magnitud de variación y permanece constante la duración, se produce un cambio más abrupto y la sensación de un movimiento continuo se pierde. Así, modificando ambas variables permite jugar con la forma en que vemos a la realidad, con lo que se pueden generar vistas diferentes que, a su vez, pueden facilitar nuevos conocimientos. Finalmente, la tercera variable dinámica según DiBiase et al. (Ídem) es el orden. El orden permite distinguir entre animaciones temporales, siguiendo un orden cronológico, y animaciones non-temporales, siguiendo algún orden atributivo (por ejemplo una animación, que muestra primero los sismos más fuertes de una región y a continuación los sismos en magnitud decreciente).

Las tres variables dinámicas presentadas se combinan con las variables estáticas de manera múltiple. Así, la animación implica para la cartografía una multiplicación de opciones de visualización. La Figura 4 demuestra estas posibilidades de combinación, utilizando nuevamente el ejemplo de un símbolo para un huracán. Aquí la combinación entre variables visuales estáticas y las tres variables dinámicas da el efecto de una rotación continua (Figura 4a), mientras una modificación en la variable duración produce una rotación acelerada (en función de diferentes intensidades del huracán durante su paso) en Figura 4b. 
Figura 4: Combinación de variables visuales estáticas y variables dinámicas para producir efectos de rotación constante (a) y acelerada (b) de un símbolo de un huracán en función de su intensidad

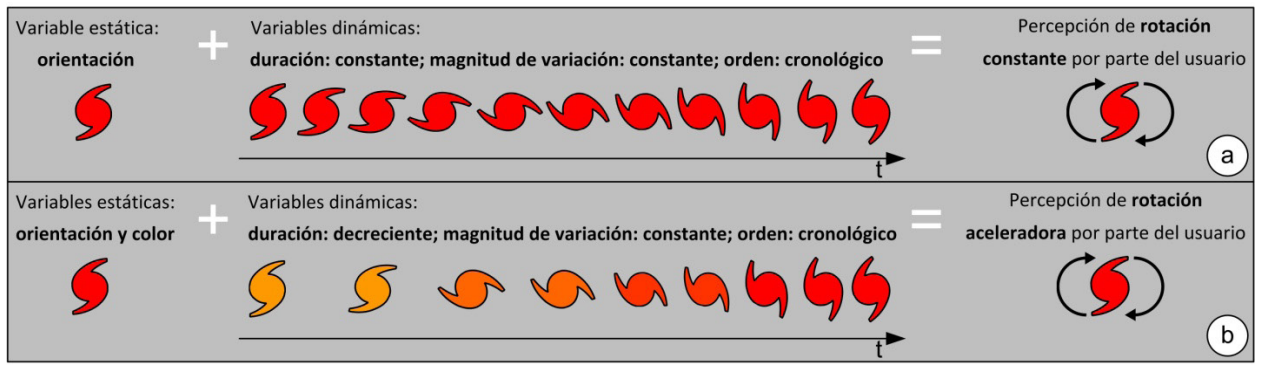

Fuente: Elaboración propia.

\section{ANIMACIÓN}

La cartografía analógica tiene una serie de opciones para visualizar cambios en el tiempo. Por ejemplo se pueden usar flechas para indicar movimientos en el tiempo, o variables visuales como el valor para visualizar cambios diacrónicos. Otra opción estática es la visualización mediante un conjunto de mapas presentadas en orden cronológico unos al lado de otros, una técnica que se conoce desde Tufte (1990) bajo el término de pequeños múltiples (small multiples). Sin embargo, como el enfoque del presente artículo se dirige a la visualización genuina del tiempo mediante el tiempo de la misma representación, el método de preferencia es la animación. Para la cartografía podemos definir la animación como una secuencia de gráficos estáticos en donde se modifican una o varias de las variables visuales en una frecuencia (normalmente entre 24 y 30 mapas por segundo) que produce la sensación de una cambio continuo de esta(s) variable(s) en el usuario (cf. Figuras 3 y 4). Por lo tanto, las animaciones, basadas en gráficos, se distinguen de las películas y los videos, conformadas por elementos fotográficos (Harrower \& Fabrikant, 2008).

Lobben (2003) propone tres criterios para categorizar los diferentes tipos de animaciones: tiempo (time), espacio (space) y tema (variable; atribute). El espacio, en esta tipología, hace referencia al mapa base de la animación, sobre el cual se visualiza el tema. Dado que nos interesa la visualización del tiempo, lo definimos como dinámico, mientras que los otros dos criterios (espacio y tema) pueden ser tanto estáticos como dinámicos. Esta condición implica que casos populares de animaciones de áreas, como por ejemplo la simulación de un sobrevuelo de un paisaje virtual, queden fuera de las siguientes consideraciones, puesto que en este tipo de animaciones no se visualiza ningún cambio temporal. La Tabla 1 muestra las 
opciones relevantes para una visualización dinámica del tiempo.

Tabla 1. Opciones para visualizar el tiempo mediante animaciones cartográficas de primero, segundo y tercer orden con los criterios de tiempo, tema y espacio

\begin{tabular}{|c|c|c|c|}
\hline \multirow{2}{*}{$\begin{array}{c}\text { Orden de la } \\
\text { animación }\end{array}$} & \multicolumn{3}{|c|}{ Criterios dinámicos de la animación } \\
\hline \multirow{2}{*}{ Primero } & Tiempo & Tema & Espacio \\
\hline Segundo & $\mathrm{x}$ & & \\
\cline { 2 - 4 } & $\mathrm{x}$ & $\mathrm{x}$ & $\mathrm{x}$ \\
\hline Tercero & $\mathrm{x}$ & $\mathrm{x}$ & $\mathrm{x}$ \\
\hline
\end{tabular}

Fuente: Elaboración propia.

La Tabla 1 da una visión general de cómo podemos crear animaciones cartográficas con complejidad ascendente. Cabe mencionar que la terminología que usamos aquí también refleja modificaciones críticas de la tipología propuesta por Lobben (Ídem), por lo cual se introduce una denominación nueva que habla de animaciones temporales de primer, segundo y tercer orden.

Animaciones temporales de primer orden $\left(\mathrm{A}^{\top 1}\right)$ se definen por la visualización dinámica del tiempo en un espacio estático con temas visualizados mediante variables estáticas. Ejemplo típico de una $\mathrm{A}^{\top 1}$ sería un mapa que visualiza los epicentros de sismos de cierta área en un orden cronológico, de tal manera que los epicentros aparecen consecutivamente en un mapa base estático en función del periodo representado. Como la calidad de las variables que representan el tema (en el ejemplo los epicentros) no cambia durante la animación de manera dinámica, la variable tema se considera como estática; por lo que la variable dinámica que queda es el tiempo en que los símbolos estáticos aparecen en el mapa base.

Figura 5. Tres escenas del mapa de epicentros de sismos en Jalisco

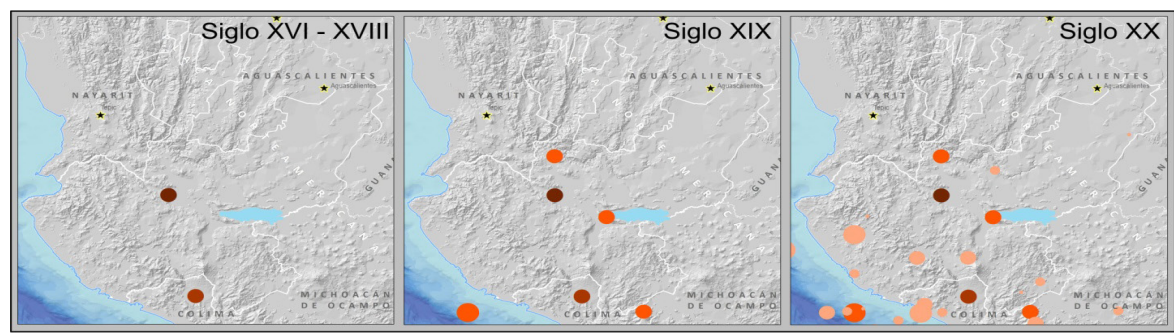

Fuente: Elaboración propia, con base en Valdivia Ornelas \& Castillo Aja (en prensa) 
En las animaciones temporales de segundo orden (AT2) se modifica un segundo criterio de manera dinámica, que puede ser ya sea el espacio del mapa base o el tema. Esta modificación tiene que realizarse de tal manera que el usuario la perciba como un cambio continuo (mientras un cambio discreto resultaría otra vez en una AT1). La Figura 8 da un ejemplo de una AT2 con base en una visualización de un huracán, donde el símbolo del huracán (el tema $(T)$ ) se modifica de manera dinámica con respecto a su posición, color, tamaño y orientación en función del evento representado. Si el segundo criterio de la AT2 no es el tema sino el espacio (E) del mapa base se habla de una (AT2-E). Un ejemplo seria una animación que muestra el movimiento de las placas tectónicas en el pasado o futuro (ver Figura 6.).

Sin embargo, animaciones del tipo AT2-E son casos especiales donde el mapa base representa espacio y tema al mismo tiempo.

Figura 6. Tres escenas de una animación del movimiento de las placas tectónicas en los próximos 100 millones de años (ma) -ejemplo de una animación temporal de segundo orden con espacio dinámico (AT2-E)

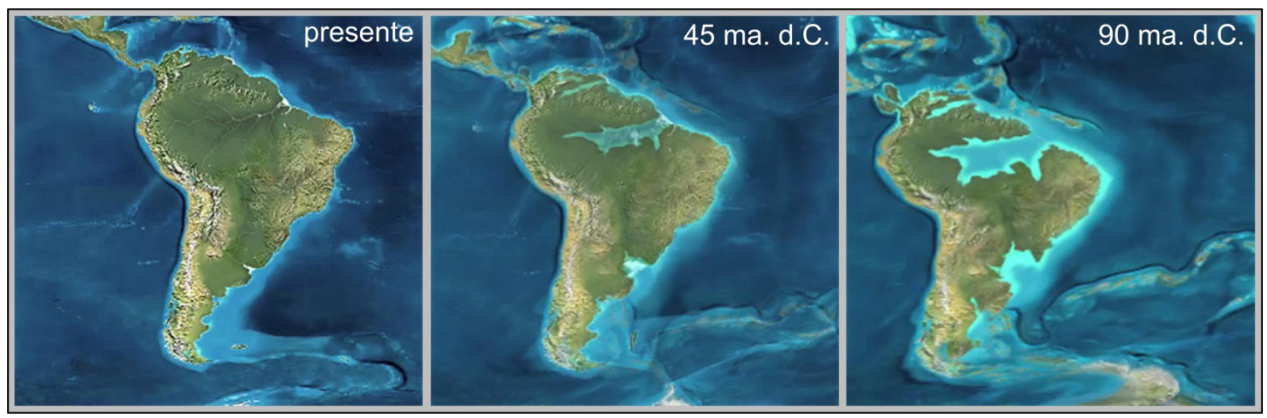

Fuente: Elaboración propia con base en datos del Departamento de Geografía de la Universidad de Viena.

El caso más complejo lo constituyen las animaciones temporales de tercer orden $\left(A^{\top 3}\right)$ donde los tres criterios mencionados tienen carácter dinámico, el resultado son animaciones donde una visualización del tiempo y del tema corre en paralelo a una modificación del mapa base, como aquellas que utilizan el zoom y el paneo. 


\section{LA VISUALIZACIÓN EN DEL TIEMPO EN LOS SIG —EL EJEMPLO DE}

\section{ARCGIS}

Como un análisis profundo de las herramientas de los diferentes SIG para visualizar el tiempo de manera dinámica excede el marco del presente trabajo, sólo se presentará a manera de ejemplo la versión más actual (10.1) del software ESRI ArcGIS, con una cuota de mercado estimada entre 40\% - 70\% el líder del mercado en nivel global. A pesar de que este software ofrece, según nuestra experiencia, la mayor cantidad de herramientas para realizar animaciones dentro del mismo SIG, aplican las siguientes consideraciones también a herramientas comparables de otros SIG, por ejemplo el time manager de QuantumGIS.

- ESRI define los tipos de animaciones que se pueden crear en ArcMap, ArcScene y ArcGlobe de la siguiente manera (ArcGIS Resource Center, 2012):

- Navegar por la visualización (zoom y desplazamiento panorámico en ArcMap, o navegar en ArcGlobe o ArcScene).

- Animar la transparencia o visibilidad de capas.

- Mover la cámara o la vista de mapa a lo largo de una ruta.

- Mover una capa a lo largo de una ruta (sólo está disponible en ArcScene).

- Animar datos a través del tiempo mientras se mueve la cámara.

- Cambiar el color de fondo, la iluminación o la exageración vertical de una escena (sólo ArcScene).

Dentro de esta lista de opciones para crear "animaciones" en ArcGIS sólo el punto cinco implica una visualización del tiempo. El punto seis hace referencia al cambio de la iluminación del paisaje con el movimiento del sol durante un día, que es por sí una animación del tiempo; sin embargo, esta herramienta no permite animaciones temporales de otras variables, por lo cual lo tratamos como caso especial que no necesita de más explicaciones aquí.

Para visualizar cambios temporales de datos espaciales de una manera genuina, ArcGIS ofrece dos opciones: El control deslizante de tiempo y la barra de herramientas de animación para crear una animación basada en el tiempo. Ambas herramientas requieren información temporal para la entidad (feature), que se tiene que definir y habilitar en las propiedades de capa. Teniendo disponible esta información temporal se puede usar por un lado el control deslizante de tiempo para estructurar (por ejemplo acortar o alargar la extensión de tiempo) esta información y visualizarla en el mismo software o exportarla a un formato de video. Como el

\footnotetext{
${ }^{1}$ Otros SIG con herramientas que se dedican más al análisis temporal que a la visualización dinámica del tiempo, como por ejemplo el Earth Trends Modeler de Idrisi, no son tema de este artículo.
} 
deslizante de tiempo no permite cambios dinámicos en el mapa base, las anima-

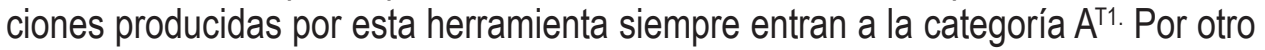
lado, ArcGIS dispone de un administrador de animaciones, con el cual se pueden integrar datos temporales en animaciones de la vista.

Comparamos entonces las opciones que ArcGIS actualmente da para visualizar el tiempo con los diferentes tipos de animaciones de la Tabla 1, queda demostrado que este software en particular, pero también los SIG en general, permiten visualizaciones dinámicas del tiempo el del espacio, mientras animaciones dinámicas del tema todavía no se pueden visualizar.

\section{LAANIMACIÓN CARTOGRÁFICA FUERA DE LOS SIG}

Además de las opciones mencionadas anteriormente sobre cómo hacer animaciones dentro de los SIG, existe la posibilidad de posprocesar datos preparados dentro de un SIG para crear visualizaciones dinámicas mediante software adicional. Un ejemplo basado en servidor lo brinda Microsoft Silverlight, que es una estructura de desarrollo para aplicaciones web que también permite la programación de aplicaciones dinámicas e interactivas. La interfaz de programación de aplicaciones (API por sus siglas en ingles) de ArcGIS para Silverlight permite la integración de servicios de ArcGIS Server en aplicaciones de Silverlight. Para aquellos SIG que no se basan en un servidor web (e.g. ArcMap) está la alternativa de exportar datos espaciales del SIG en un formato vectorial, que pueden ser importados y trabajados, por ejemplo, en Adobe Flash.

A continuación se presentará esta opción de manera ejemplar mediante de una animación del fenómeno meteorológico de los ciclones tropicales, que se realizó como prototipo para la actualización del Atlas de riesgos por fenómenos naturales del estado de Jalisco (México). La animación se sirve de una base de datos mantenida por la Administración Nacional Oceánica y Atmosférica (NOAA, por sus siglas en inglés), que ofrece actualmente datos de casi 300,000 ciclones tropicales a nivel mundial. Esos datos fueron filtrados, fijados a escala y integrados en un mapa base en un SIG. Después se hizo una exportación de este mapa base al formato vectorial de Adobe Illustrator (.ai), donde se armaron los datos espaciales con los elementos de interfaz gráfica de usuario que permite una navegación personalizada para los usuarios de la animación. Finalmente, se importaron los datos elaborados en Adobe Illustrator al Adobe Flash, donde se programaron las animaciones de las rutas de diferentes huracanes relevantes para el área del estado de Jalisco. Las variables visuales utilizadas fueron el tamaño, el color y la 
orientación. Aplicando técnicas de tweening ${ }^{2}$, estas variables se modifican en la animación continuamente en función de la intensidad del huracán; se complementa indicando el área afectada por el huracán, también de manera dinámica sobre el mapa base (Figura 7).

Figura 7: Escenas del prototipo de la animación del huracán Kenna, llegando a las costas de Jalisco el 25 de octubre 2002

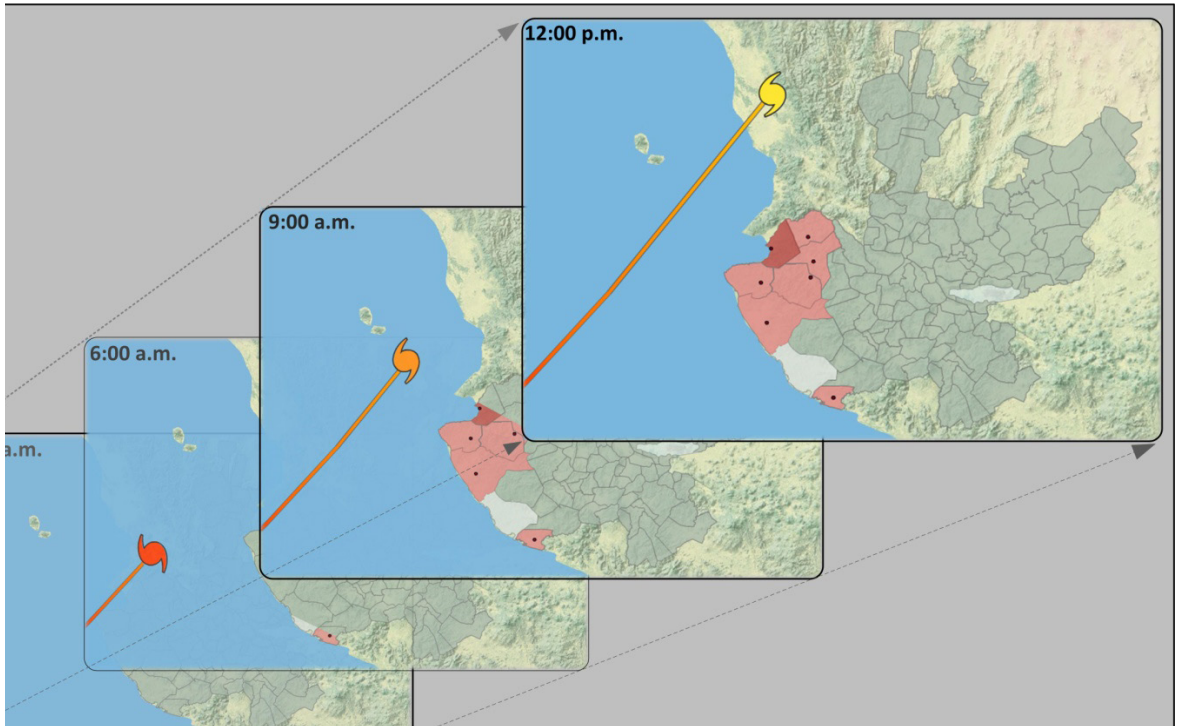

Fuente: Elaboración propia a base de Segundo \& Hruby (2012)

Si ahora comparamos las opciones de programas como Adobe Flash con la Tabla 1, podemos constar que la animación cartográfica fuera de los SIG nos permite visualizar no solo el tiempo y el espacio sino también el tema del mapa de manera dinámica en términos de animaciones de tipo $A^{\top 2}$ y $A^{T 3}$.

\section{CONCLUSIONES}

Desde que Wood \& Fels (1992) criticaron a la cartografía por tratar el tiempo como una dimensión escondida, las opciones de los SIG para visualizar datos temporales han crecido de manera constante. Los SIG de punta trabajan tanto información temporal como espacial. Sin embargo, a los SIG aún les falta la capacidad para visualizar el tema del mapa de manera dinámica. Para superar estas

${ }^{2}$ Tweening se refiere a técnicas de interpolación automatizada entre gráficos (cf. Batterby \& Goldsberry, 2010) 
limitaciones se puede extender el proceso de la elaboración cartográfica, incluyendo software y estándares especializados, como por ejemplo Adobe Flash, Microsoft Silverlight o HTML5, que permiten un posprocesamiento de datos espaciales preparados en un SIG. Usando técnicas como tweening y morphing ${ }^{3}$ y considerando las variables dinámicas de DiBiase et al. (1992) se pueden modificar las variables visuales de Bertin de manera continua para lograr animaciones de tercer orden. Además, estos programas permiten agregar elementos multimedia y funciones de interactividad y navegación a los mapas - factores importantes tomando en cuenta que una animación, por su carácter dinámico, es más compleja en su uso y demanda un control adecuado por parte del usuario.

Cabe notar que el desarrollo de teorías adecuadas en el área de cartografía y SIG no mantuvo el mismo ritmo de avance que el desarrollo tecnológico de las últimas décadas (Harrower \& Fabrikant, 2008); por lo que no está suficientemente investigado, cuáles serían las ventajas de utilizar qué tipo de temas y con cuáles tipos de animaciones; tampoco existe una terminología elaborada para diferenciar entre tipos de animaciones de manera consistente. Por lo tanto, se ha presentado en apego critico a Lobben (2003) una tipología de animaciones del tiempo y a la vez una aplicación ejemplar de esta tipología para describir las posibilidades de visualizar el tiempo dentro y fuera de los SIG den manera dinámica. Sin embargo, el tema de las visualizaciones de tiempo en la cartografía amerita más investigación para comprobar tanto la adecuación de la tipología propuesta en este artículo, como la utilidad de las animaciones para los fines de la cartografía.

\section{BIBLIOGRAFÍA}

- ArcGIS Resource Center (2012). “¿Qué es una animación?”, [consulta: 2404-2013]. Disponible en: http://resources.arcgis.com/es/help/main/10.1/index. html\#/na/000900000001000000/

- Bertin, J. (1967). Sémiologie Graphique. Les diagrammes, les réseaux, les cartes. Paris: Gauthier-Villars.

- Battersby, S. y Goldsberry K.P. (2010). "Considerations in Design of Transition Behaviors for Dynamic Thematic Maps", Cartographic Perspectives, vol. 65,

\footnotetext{
${ }^{3}$ Morphing se refiere a técnicas de interpolación automatizada para trasladar la forma de una imagen/ de un gráfico a la forma de otra imagen/otro gráfico. El tweening forma normalmente un paso en el proceso del morphing. Figura 6 da un ejemplo de esta técnica.
} 
pp. 16-32.

- DiBiase, D., MacEachren, A.M., Krygier, J.B. y C.R. Reeves (1992). "Animation and the Role of Map Design in Scientific Visualization", Cartography and Geographic Information Systems, vol. 19 (4), pp. 201-214, 265-266.

- Dorling, D. y S. Openshaw (1992). "Using computer animation to visualize space-time patterns", Environment and Planning B: Planning and Design, vol. 19, pp. 639-650.

- Frank, A. (1994). "Different types of 'times' in GIS", en Egenhofer, M., y R. Golledge: Spatial and Temporal Reasoning in Geographic Information Systems. New York: Oxford Press. pp. 40-62.

- Griffin, A. L., MacEachren, A.M., Hardisty, F, Steiner, E y B. Li (2006). "A Comparison of Animated Maps with Static Small-Multiple Maps for Visually Identifying Space-Time Clusters", Annals of the Association of American Geographers, vol. 96 (4), pp. 740-753.

- Harrower, M. y S.I. Fabrikant (2008). "The role of map animation in geographic visualization." en Dodge, M., McDerby, M. y M. Turner: Geographic Visualization: Concepts, Tools and Applications. Chichester, Wiley. pp. 49-65.

- Kraak, M.-J. y F. Ormeling (2010). Cartography: Visualization of Geospatial Data. Pearson Education, Harlow.

- Lobben, A. (2003). "Classification and Application of Cartographic Animation", The Professional Geographer, vol. 55 (3), pp. 318-328.

- MacEachren, M. (2004). How maps work: representation, visualization, and design. New York: The Guilford Press.

- Mundy, B. E. (1996). The Mapping of New Spain. Chicago: The University of Chicago Press.

- Ott, T., y F. Swiaczny (2001). "Integrating time in Geographic Information Systems.", en Ott, T., y F. Swiaczny: Time-Integrative Geographic Information Systems. Berln, Springer. pp. 55-75.

- Segundo Metay, P. \& Hruby F. (2012). "Visualización del tiempo mediante animaciones cartográficas" en Memorias: VIII Reunión Nacional de Geomorfología 
[consulta: 24-04-2013]. Disponible en: http://www.igeograf.unam.mx/web/sigg/ docs/pdfs/eventos/conferencias/MEMORIAS.pdf

- Tobler, W. (1970). "A computer movie simulating urban growth in the Detroit region”, Economic Geography, vol. 46, pp. 234-240.

- Tufte, E. (1990). Envisioning Information, Cheshire: Graphics Press.

- Valdivia Ornelas, L. y M.d.R. Castillo Aja. (En prensa). "Impacto de los desastres naturales en la historia reciente del Estado". Guadalajara: Editorial Universidad de Guadalajara.

- Wood, D. y J. Fels (1992). The Power of Maps, The Guilford Press, New York. 